\title{
Hyaluronan induces odontoblastic differentiation of dental pulp stem cells via CD44
}

\author{
Naoki Umemura ${ }^{1,2^{*}}$ (D, Emika Ohkoshi ${ }^{3}$, Masamichi Tajima ${ }^{1}$, Hirotaka Kikuchi ${ }^{4}$, Tadashi Katayama ${ }^{5}$ \\ and Hiroshi Sakagami ${ }^{1}$
}

\begin{abstract}
Background: Dental pulp tissue contains many undifferentiated mesenchymal cells, which retain the ability to differentiate into mature cells. Induced pluripotent stem cells have been developed from various cell sources, including dental pulp-derived stem cells, and evaluated for potential application to regenerative therapy. Dental pulp tissues overexpress CD44, a cell-adhesion factor involved in the induction of mineralization. In this study, we investigated the effects of hyaluronan — a known CD44 ligand—on dental pulp stem cells (DPSCs).
\end{abstract}

Methods: DPSC CD44 expression was analyzed using immunofluorescence staining, flow cytometry, and western blotting. Cell proliferation was evaluated using the 3-(4,5-dimethylthiazol-2-yl)-2,5-diphenyltetrazolium bromide assay. Effects of hyaluronan on the cell cycle were analyzed by flow cytometry. Alkaline phosphatase activity was employed as marker of mineralization and measured by fluorometric quantification and western blotting. Bone morphogenetic protein (BMP)-2, BMP-4, dentin sialophosphoprotein (DSPP), and dentin matrix acidic phosphoprotein 1 (DMP-1) levels were measured using real-time polymerase chain reaction. Odontoblastic differentiation and the close cell signaling examination of DPSC differentiation were determined using western blotting.

Results: Hyaluronan induced expression of the odontoblastic differentiation markers DMP-1 and DSPP. Moreover, the odontoblastic differentiation induced by hyaluronan was mediated by CD44—but not by Akt, Smad1 or MAPK signaling.

Conclusions: Our results indicate that hyaluronan induces odontoblastic differentiation of DPSCs via CD44. This suggests that hyaluronan plays a crucial role in the induction of odontoblastic differentiation from DPSCs. Our findings may aid the development of new, inexpensive, and effective conservative treatments for dental pulp repair.

Keywords: Dental pulp calcification, Bone mineralization, DMP-1 protein, DSPP protein, Dental pulp capping, Smad1 protein

\section{Background}

Dental pulp cells have the capacity to differentiate into odontoblasts. Dental damage caused by oral cavities, periodontal disease, or mechanical trauma induces the formation of reparative dentin, a poorly organized mineralized matrix that serves as a protective barrier to the dental pulp [1].

\footnotetext{
* Correspondence: umemura@dent.asahi-u.ac.jp

'Division of Pharmacology, Department of Diagnostic and Therapeutic

Sciences, Meikai University School of Dentistry, 1-1 Keyakidai, Sakado, Saitama 350-0283, Japan

2Department of Oral Biochemistry, Asahi University School of Dentistry, Gifu

501-0296, Japan

Full list of author information is available at the end of the article
}

Dental pulp stem cells (DPSCs) are present in human dental pulp, even in adult pulp, as clonogenic and highly proliferative cells obtained after enzymatic disaggregation [2]. These cells harbor the characteristics of plastic adherence and express stem cell markers such as CD29, CD90, CD44, and CD146 [2]. Additionally, DPSCs express transcription factors expressed by embryonic stem cells, including Oct-4, Sox-2 and Nanog [3, 4]. Numerous researchers have since shown that DPSCs retain the capacity for both self-renewal and multiple cell lineage differentiation [5, 6] and can be stimulated, under specific conditions, to differentiate into various cell types such as adipocytes, myoblasts, neurons, 
chondrocytes, odontoblasts and osteoblasts both in vitro and in vivo [7-9]. Animal studies have also revealed great potential for DPSCs in the repair and regeneration of various tissues, including bone [10], muscle [3] and teeth [11].

Odontoblasts, especially those in the root ends of immature teeth, express CD44, which is strongly expressed by cells undergoing mineralization, such as ameloblasts, odontoblasts and osteoblasts in calcifying areas [12]. CD44 functions as an adhesion molecule and is a broadly distributed type I transmembrane glycoprotein receptor for the glycosaminoglycan hyaluronan (HA) $[13,14]$. However, the effects of HA stimulation of CD44 on DPSCs remain unknown. In this study, we investigated the effect of HA on DPSCs.

\section{Methods}

\section{Reagents and cell culture}

Hyaluronic acid sodium salt (CAS number 9067-32-7) was purchased from Nacalai Tesque Co. (Kyoto, Japan). This reagent was slowly dissolved in double-distilled water to a final concentration of $10 \mathrm{mg} / \mathrm{mL}(1 \%)$. HA was further diluted in culture medium to required concentrations prior to use in cell culture experiments. Dulbecco's modified Eagle's medium (DMEM) was purchased from Invitrogen (Carlsbad, CA, USA). Fetal bovine serum (FBS) was purchased from Nichirei Bioscience (Tokyo, Japan).

Human DPSCs were obtained from AllCells LLC (Emeryville, CA, USA). Cell cultures were maintained in DMEM supplemented with $10 \%$ FBS and antibiotics (100 U/mL penicillin and $100 \mu \mathrm{g} / \mathrm{mL}$ streptomycin) at $37{ }^{\circ} \mathrm{C}$ in a humidified atmosphere containing $5 \% \mathrm{CO}_{2}$. The passage numbers were limited at $2-5$ to avoid cell deterioration.

\section{Immunofluorescence}

Monolayers of DPSCs were cultured with DMEM containing $10 \%$ FBS for $48 \mathrm{~h}$ in four-well covered glass chamber slides. After two washes with phosphatebuffered saline (PBS) containing $1 \%$ bovine serum albumin (Sigma-Aldrich, St. Louis, MO, USA), cell surface Fc receptors were blocked with immunoglobulin G (IgG) (Santa Cruz Biotechnology Inc., Dallas, TX, USA) on ice for $15 \mathrm{~min}$. The cells were then stained for $30 \mathrm{~min}$ at $37{ }^{\circ} \mathrm{C}$ with a $1: 100$ dilution of a fluorescein isothiocyanate (FITC)-conjugated anti-CD44 monoclonal antibody (BD Biosciences, Franklin Lakes, NJ, USA) or an isotype-matched FITC-conjugated IgG control antibody (BD Biosciences). After washing, the cells were analyzed using an ECLIPSE TS100-F microscope equipped with an Intensilight C-HGFIE illumination system (Nikon Co., Ltd., Tochigi, Japan). Digital images were processed with NIS Elements BR3.2 imaging software (Nikon Co.,
Ltd.) and Adobe Photoshop 7.0 (Adobe Systems, San Jose, CA, USA).

\section{Evaluation of cell growth using the MTT assay}

DPSCs were seeded into 96-well microtiter plates at a density of $1 \times 10^{3}$ cells/well and allowed to adhere for $24 \mathrm{~h}$. Cell viability was assessed on a daily basis by addition of $5 \mu \mathrm{L}$ of 3-(4,5-dimethylthiazol-2-yl)-2,5-diphenyltetrazolium bromide (MTT) using a Cell Proliferation Kit I (Roche Diagnostics, Mannheim, Germany), according to the manufacturer's instructions. The number of viable cells was assessed by measuring the absorbance of the produced formazan crystals at $595 \mathrm{~nm}$ with a MultiSkan JX microplate reader and Ascent software (Thermo Labsystems, Vantaa, Finland). The measurement was performed once per day for 5 days. Cell growth was calculated relative to the value on the first day, which was set at $100 \%$.

\section{Quantification of alkaline phosphatase}

DPSCs were seeded into 24-well plates at a density of $5 \times 10^{4}$ cells/well, and incubated with HA $(1-20 \mu \mathrm{g} / \mathrm{mL})$ for 1 week. The cells were then harvested as cell lysates using a SensoLyte ${ }^{\circledR}$ FDP Alkaline Phosphatase Assay Kit (AnaSpec, San Jose, CA, USA), which uses a fluorogenic assay to determine alkaline phosphatase (ALP) activity. The assay was performed according to the manufacturer's instructions, and fluorescence signals were measured with SpectraFluor plus XFluor4 software (Tecan Japan Co., Ltd., Kawasaki, Japan).

\section{Flow cytometry analysis}

For analysis of CD44-positive cell surface antigen expression, untreated and HA-treated DPSCs were harvested by trypsinization, washed with PBS, centrifuged into cell pellets and resuspended in fluorescence-activated cell sorting (FACS) buffer (PBS containing $0.5 \%$ bovine serum albumin). The cells were stained for $30 \mathrm{~min}$ at $4{ }^{\circ} \mathrm{C}$ with a FITC-conjugated anti-human CD44 antibody (BD Biosciences, San Jose, CA, USA) or an isotype-matched FITC-conjugated IgG control antibody (BD Biosciences). Flow cytometry was performed using an EPICS Altra flow cytometer (Beckman Coulter, Brea, CA, USA) and the data were analyzed using Expo-3 v1.2B software (Beckman Coulter).

For cell cycle analysis, the cell cycle distribution of cells was assayed after $48 \mathrm{~h}$ by using flow cytometry to measure the DNA content of nuclei labeled with PI according to the manufacturer's instructions (BD Pharmingen, BD BioSciences). Data acquisition and analysis were performed using an EC800 flow cytometer (Sony Biotechnology, Tokyo, Japan) with EC800 analysis software (Sony Biotechnology). 


\section{Immunoblot analysis}

Whole-cell extracts from DPSCs were obtained using a lysis buffer (10× RIPA buffer; Cell Signaling Technology, Beverly, MA, USA) supplemented with $1 \mathrm{mM}$ phenylmethanesulfonyl fluoride plus one tablet of protease inhibitor cocktail (Complete, ethylenediaminetetraacetic acid (EDTA)-free; Roche Diagnostics GmbH, Mannheim, Germany). Aliquots of cell lysates (50 $\mu$ g protein) were separated by $8 \%$ or $12 \%$ sodium dodecyl sulfate polyacrylamide gel electrophoresis and electroblotted onto polyvinylidene difluoride membranes. The membranes were probed with primary antibodies, comprising anti-CD44 mouse monoclonal antibody, anti-phospho-Akt rabbit monoclonal antibody, anti-Akt rabbit monoclonal antibody, anti-phospho-GSK3 $\beta$ rabbit monoclonal antibody, anti-phospho-Smad1 rabbit monoclonal antibody, anti-Smad1 rabbit monoclonal antibody, anti-phospho- $\beta$ catenin (Ser552) rabbit monoclonal antibody, antiphospho- $\beta$-catenin (Ser675) rabbit monoclonal antibody, anti- $\beta$-catenin rabbit monoclonal antibody, anti-phosphop44/42 MAPK rabbit monoclonal antibody (all from Cell Signaling Technology, Danvers, MA, USA), anti-dentin sialophosphoprotein (DSPP) mouse monoclonal antibody (Santa Cruz Biotechnology Inc.), anti-dentin matrix protein-1 (DMP-1) rabbit polyclonal antibody, anti-ALP rabbit monoclonal antibody (both from Abcam PLC, Cambridge, UK), and anti-beta-actin antibody (Sigma-Aldrich) at the dilutions recommended by the manufacturers. Signals were detected using corresponding peroxidaseconjugated secondary antibodies (anti-rabbit IgG antibody or anti-mouse IgG antibody; Cell Signaling Technology), and signal bands were visualized by chemoluminescence (Clarity $^{\text {mix }}$ Western ECL substrate; Bio-Rad, Hercules, CA, USA). The membranes and images were developed with a ChemoDoc $^{\text {tx }}$ Imaging System (Bio-Rad).

\section{Real-time polymerase chain reaction}

Total RNA was purified using Trizol reagent (Invitrogen Life Technologies, Carlsbad, CA, USA), and $600 \mathrm{ng}$ of total RNA was used for reverse transcription with an iScript $^{\mathrm{tm}}$ Advanced cDNA Synthesis Kit (Bio-Rad). For real-time polymerase chain reaction analysis, $1 \mu \mathrm{L}$ of cDNA sample at 1:20 dilution, $1 \mu \mathrm{L}$ each of forward and reverse primers (final, $500 \mathrm{nM}$ ), $7 \mu \mathrm{L}$ of nuclease-free water, and $10 \mu \mathrm{L}$ of SsoAdvanced SYBR Green Supermix (Bio-Rad) were used. The following primers were used: DMP-1 (GenBank ID: NM_004407.3) forward primer 5'-CCTGAGGATGAGAACAGCTCCA-3' and reverse primer 5'-GATCTGCTGCTGTCTTGAGAGTCAC-3'; DSPP (GenBank ID: NM_014208.3) forward primer 5'CCAGAGCAAGTCTGGTAACGGTAA-3' and reverse primer 5'-GTCACTGCCTTCACTGTCACTGTC-3'; bone morphogenetic protein (BMP)-2 (GenBank ID: NM_001200.2) forward primer 5'-GGAACGGACATT
CGGTCCT-3' and reverse primer 5'-GGAAGCAGCAA CGCTAGAAG-3'; BMP-4 (GenBank ID: NM_001202.3) forward primer 5'-TCACTGCAACCGTTCAGAGGTC3 ' and reverse primer 5'-CCAATCTTGAACAAACTTG CTGGA-3'; and GAPDH (GenBank ID: NM_002046.5) forward primer 5'-GCACCGTCAAGGCTGAGAAC-3' and reverse primer $5^{\prime}$-TGGTGAAGACGCCAGTGGA3 . Reaction conditions were one 5 -min cycle at $95{ }^{\circ} \mathrm{C}$, followed by 45 cycles of $95{ }^{\circ} \mathrm{C}$ for $10 \mathrm{~s}$ and $72{ }^{\circ} \mathrm{C}$ for $10 \mathrm{~s}$. The reactions and relative quantification analyses were performed using a LightCycler 480 instrument (Roche Diagnostics, Indianapolis, IN, USA).

\section{Signal blocking assays}

DMH-1, a Smad1/5 inhibitor and SCH772984, a novel ERK1/ 2-specific inhibitor were purchased from Selleckchem.com (http://www.selleckchem.com/). LY294002, an Akt inhibitor, was purchased from Cell Signaling Technology. An antiCD44 monoclonal antibody (Clone A3D8; Sigma-Aldrich) was used in neutralization assays.

DPSCs were pretreated with the inhibitors or CD44-blocking antibody for $30 \mathrm{~min}$ before stimulation with HA $(10 \mu \mathrm{g} / \mathrm{mL})$. After $30 \mathrm{~min}$ of stimulation, the cells were harvested to investigate the inhibition of phosphorylation for several signaling molecules. After $24 \mathrm{~h}$, the odontoblastic differentiation markers DMP1 and DSPP were evaluated by immunoblotting.

\section{Statistical analysis}

Data are presented as the mean $\pm S D$ and evaluated using one-way analysis of variance followed by Dunnett's multiple comparison. Values of $P<0.05$ were accepted as statistically significant.

\section{Results}

\section{Expression of CD44 in DPSCs}

Most CD44 antigenicity in dental pulp tissue is present in the incomplete region of the roots [12]. However, whether these CD44-expressing cells are DPSCs is unknown. Consequently, we identified CD44 expression on the DPSC cell surface (Fig. 1a, upper panels). Flow cytometry revealed a high proportion of CD44-positive cells (approximately 62 \%; Fig. 1b, c). We then investigated whether CD44 expression was altered by treatment with $\mathrm{HA}$, a known CD44 ligand [15]. We found that the number of CD44expressing cells was significantly increased from $62 \%$ to $72 \%$ at 5 min following treatment with HA, while this expression significantly decreased to $54 \%$ after $30 \mathrm{~min}$ and continued to decrease to $22 \%$ (Fig. 1c).

\section{HA induces mineralization in DPSCs}

We examined whether HA induces DPSC cell growth, and found that HA had no significant effect on the 


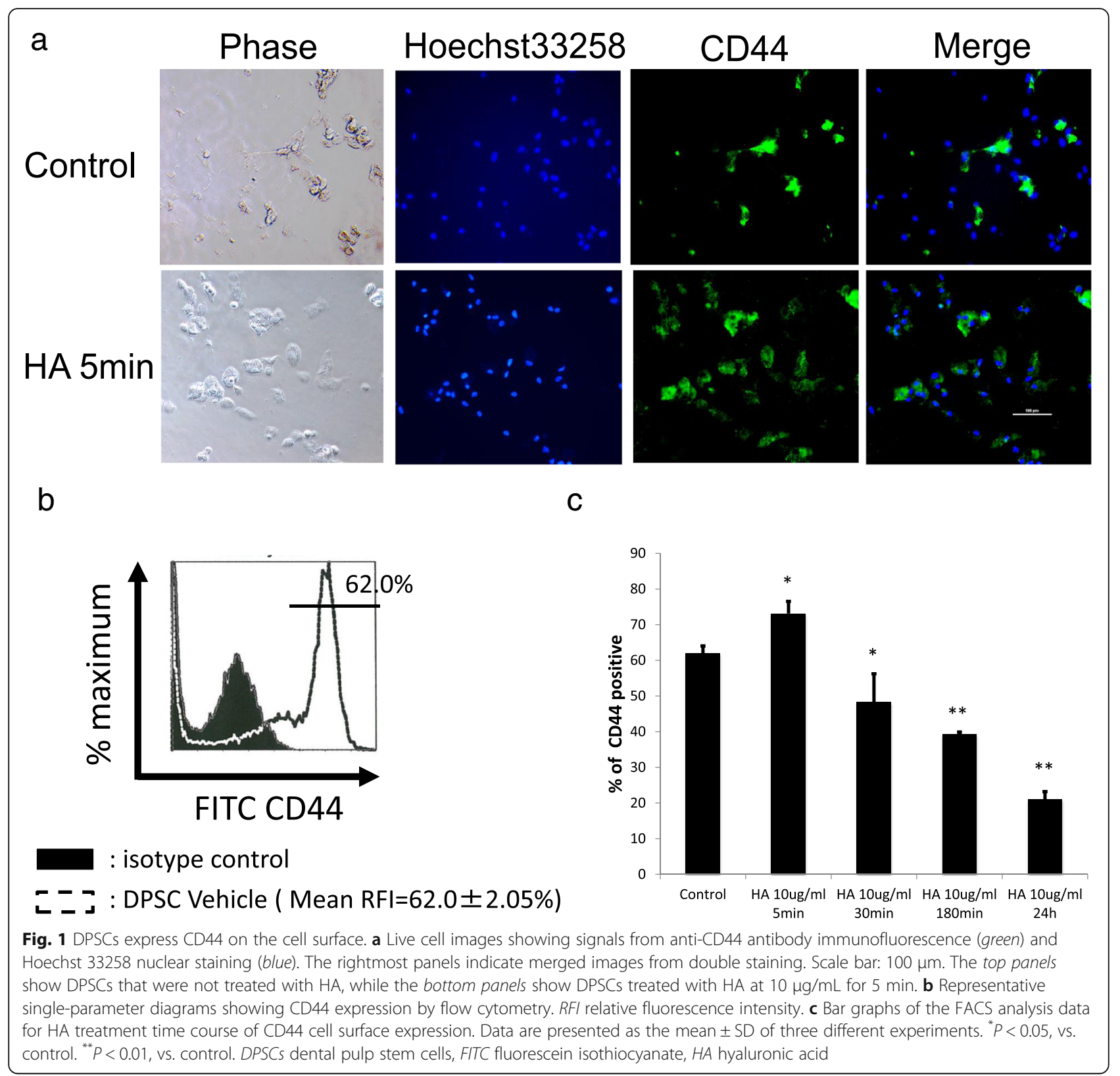

DPSC cell proliferation (Fig. 2a). Additionally, cell cycle analysis revealed that $\mathrm{HA}$ does not influence the cell cycle (Fig. $2 \mathrm{~b}$ and $\mathrm{c}$ ). We then investigated whether DPSCs were otherwise affected by treatment with HA, a known ligand of CD44. First, we considered whether DPSC mineralization was influenced by $\mathrm{HA}$, because CD44 in pulp tissue plays an important role in mineralization [12]. Therefore, we used immunoblotting to measure ALP protein levels as an indicator of mineralization $[6,16]$. ALP protein levels increased in a concentration-dependent manner when DPSCs were cultured with HA for 1 week (Fig. 2d and e). Next, we quantified the amount of ALP using a fluorogenic assay (Fig. 2e), and found similar results. These results suggest that DPSC mineralization was guided by $\mathrm{HA}$.

HA induces odontoblastic differentiation, but not osteogenic differentiation, in DPSCs

We also investigated how HA induces DPSCs to undergo mineralization, to evaluate whether HA induces odontoblastic differentiation or osteogenic differentiation. We measured the mRNA levels of BMP2 and BMP-4 as osteogenic differentiation markers $[17,18]$ and DSPP and DMP-1 as odontoblastic 


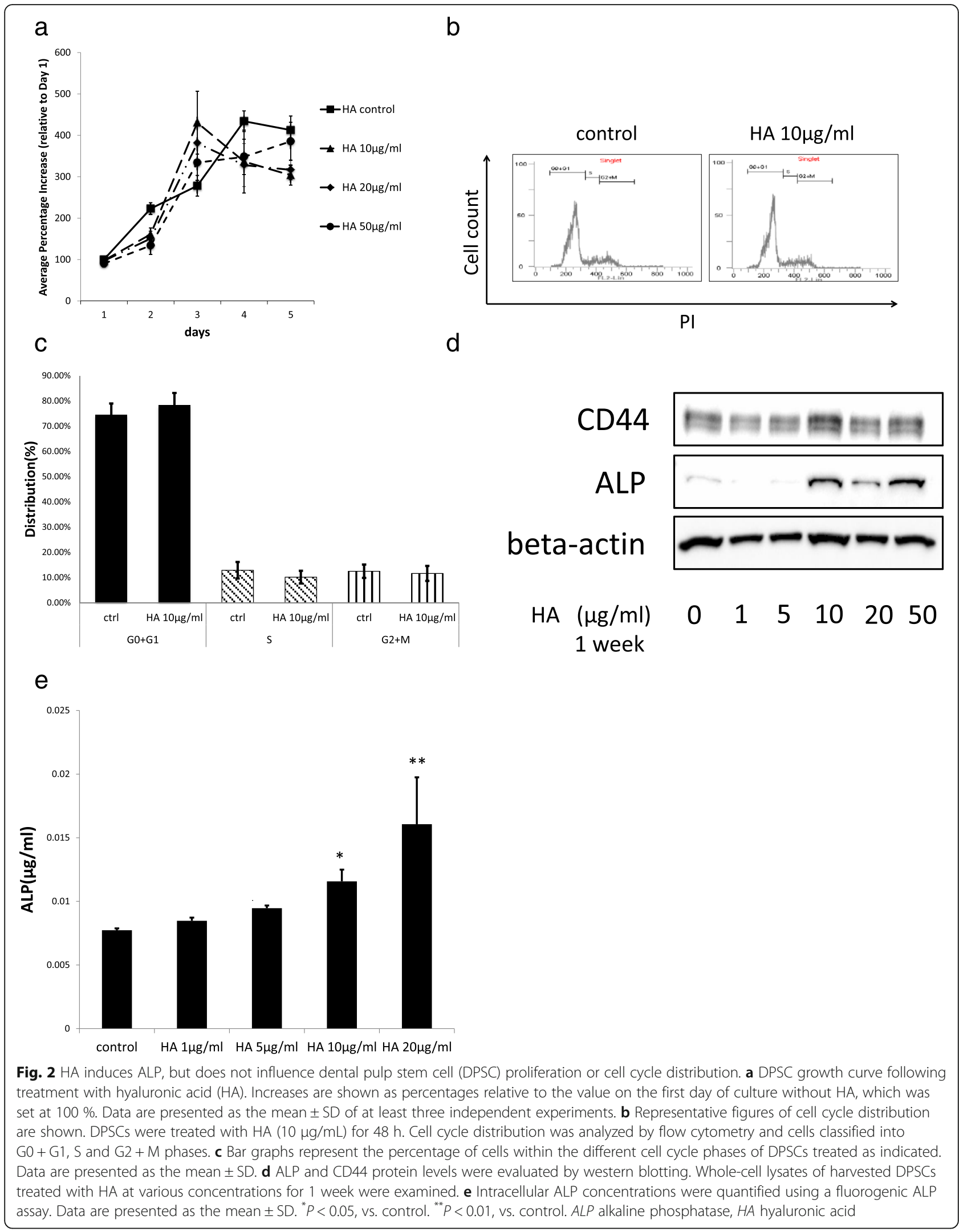


differentiation markers $[19,20]$ in DPSCs cultured with $\mathrm{HA}$ for $24 \mathrm{~h}$. We found that BMP-2 and BMP-4 mRNA levels underwent no significant changes, while DSPP and DMP-1 mRNA levels were markedly increased. The DMP-1 mRNA level increased 7.7-fold, while that of DSPP increased 6.7-fold (Fig. 3a). Additionally, we found that HA also increased DMP1 and DSPP protein levels (Fig. 3b). These results suggest that HA stimulated DPSCs toward odontoblastic differentiation.

\section{HA-induced odontoblastic differentiation does not involve Akt, Smad1 or MAPK signaling}

The above data suggest that HA stimulates DPSCs toward odontoblastic differentiation, but the underlying mechanism remains unclear. There have been few studies of signaling in DPSCs, so we next examined the types of intracellular signaling induced by HA in DPSCs on the basis of citations provided in a previous report [21]. We investigated whether HA could induce Akt phosphorylation, because the induction of differentiation by $\mathrm{HA}$ is thought to occur by activation of PI3 kinase and Akt in DPSCs [22, 23]. We found that peak levels of Akt and GSK3 $\beta$ phosphorylation occurred 30-45 min after treatment with HA (Fig. 4a), confirming that Akt signaling and downstream GSK3 $\beta$ signaling were activated in DPSCs. We then verified that DPSCs were induced to undergo differentiation into odontoblasts in response to this Akt signaling by examining whether the odontoblastic differentiation of DPSCs induced by HA stimulation was inhibited in the presence of LY294002, an Akt activation inhibitor [24]. We found that DMP-1 expression was not inhibited, although LY294002 did inhibit the Akt and GSK3 $\beta$ phosphorylation induced by HA treatment (Fig. 4b, c). We also investigated Smad signaling, as these signals are involved in osteogenic differentiation. Specifically, we examined whether HA could induce Smad signaling during odontoblastic differentiation of DPSCs. Smad1 phosphorylation reached a peak after $30-45 \mathrm{~min}$ of treatment (Fig. 5a), similar to findings for Akt. Furthermore, we investigated whether the odontoblastic differentiation mediated by HA could be inhibited by culture with DMH-1, a Smad1 phosphorylation inhibitor [25]. Although DMH-1 inhibited Smad1 phosphorylation after treatment with HA (Fig. 5b), DMP-1 expression was not inhibited (Fig. 5c).

Others have reported that HA-CD44 signaling in other cells activates Erk1/2, a mitogen-activated protein kinase (MAPK) [26, 27]. Therefore, we next investigated MAPK signaling. HA-induced DMP-1 expression was not affected by the inhibition of Erk1/ 2 phosphorylation by $\mathrm{SCH} 772984$, a novel inhibitor of Erk1/2 activation [28] (Fig. 6a-c). We also considered Wnt/beta-catenin signaling, because others have

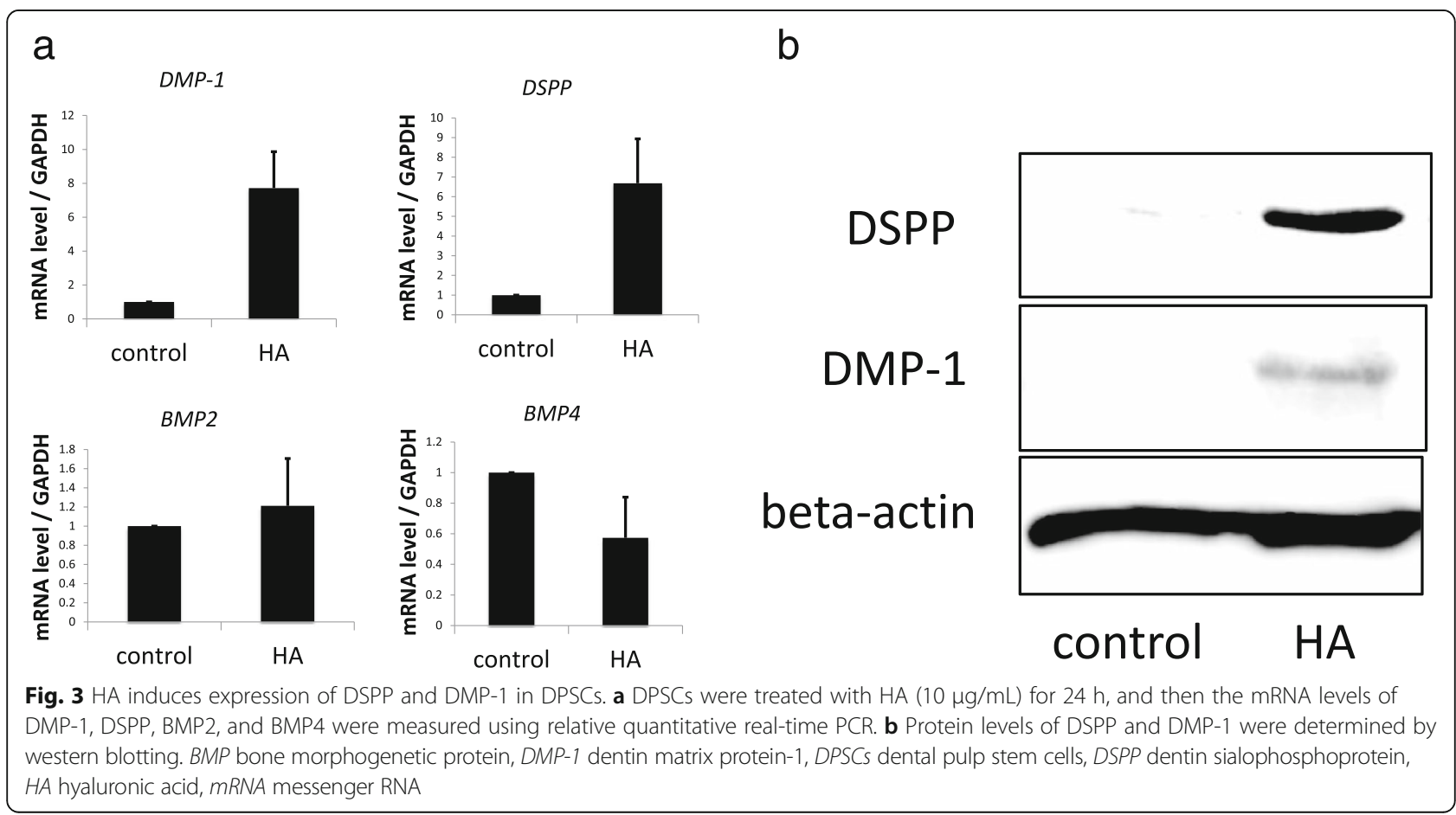


a

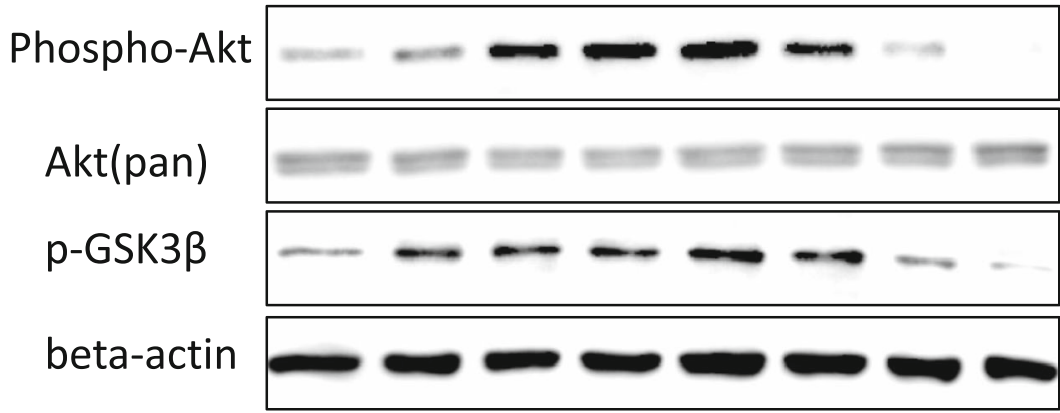

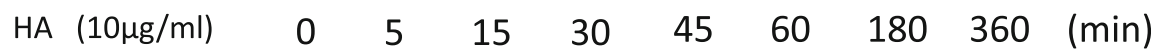

b

Phospho-Akt

p-GSK3 $\beta$

beta-actin
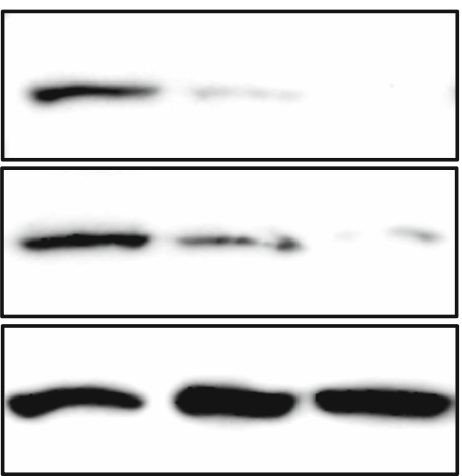

$\mathrm{HA}(10 \mu \mathrm{g} / \mathrm{ml})+\quad+$

LY294002 ( $\mu \mathrm{M}) \quad$ - $\quad 10 \quad 50$

C

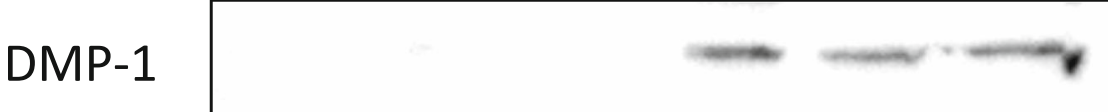

beta-actin

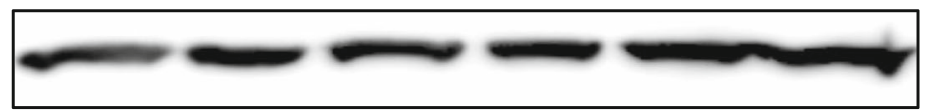

$\begin{array}{ccccccc}\mathrm{HA}(10 \mu \mathrm{g} / \mathrm{ml}) & - & - & - & + & + & + \\ \mathrm{LY} 294002(\mu \mathrm{M}) & - & 10 & 50 & - & 10 & 50\end{array}$

Fig. $4 \mathrm{HA}$ induces phosphorylation of Akt in DPSCs. a DPSCs were treated with HA (10 $\mu \mathrm{g} / \mathrm{mL})$ over a time course of 5-360 min, and then examined for

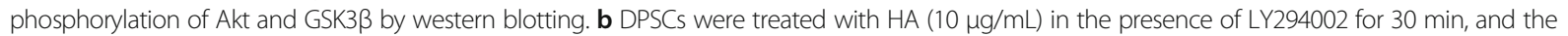
inhibition of Akt phosphorylation was validated. c Following inhibition of Akt phosphorylation by LY294002 treatment, DMP-1 expression was determined by western blotting. DMP-1 dentin matrix protein-1, HA hyaluronic acid

investigated the importance of this signaling pathway in odontogenic differentiation in recent years [29]. However, HA did not induce phosphorylation of betacatenin (Additional file 1: Figure S1). These findings indicate that although Smad, Akt and MAPK signaling were all activated by HA in DPSCs, these pathways were not involved with the odontoblastic differentiation of DPSCs. 


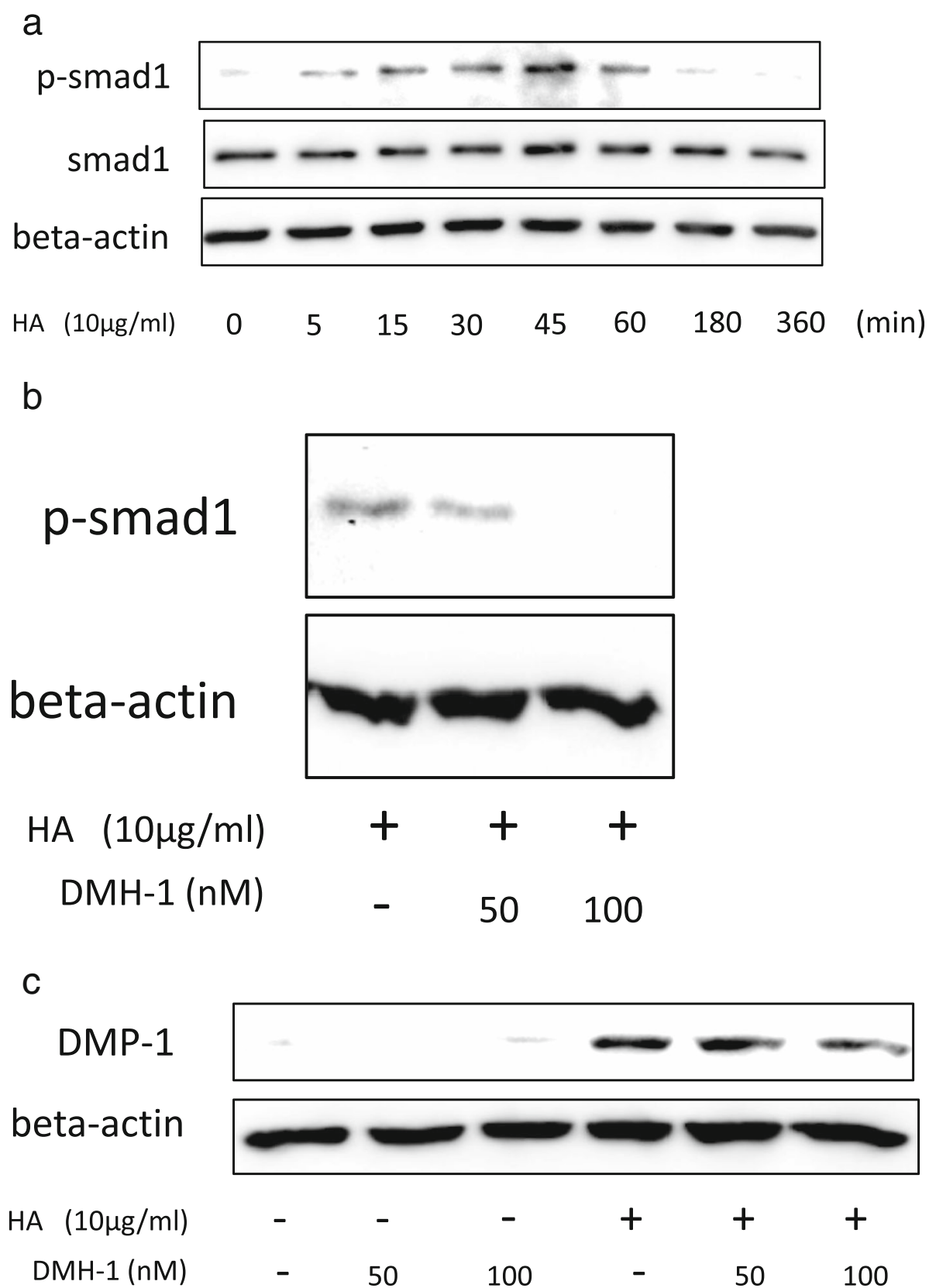

Fig. $5 \mathrm{HA}$ induces phosphorylation of Smad1 on DPSCs. a DPSCs were treated with HA (10 $\mu \mathrm{g} / \mathrm{mL})$ over a time course of 5-360 min and then examined for phosphorylation of Smad1 by western blotting. b DPSCs were treated with HA (10 $\mu \mathrm{g} / \mathrm{mL})$ in the presence of DMH-1 for $30 \mathrm{~min}$, and the inhibition of Smad1 phosphorylation was verified. c Following inhibition of Smad1 phosphorylation by DMH-1 treatment, DMP-1 expression was determined by western blotting. DMP-1 dentin matrix protein-1, HA hyaluronic acid

Odontoblastic differentiation of DPSCs induced by HA treatment involves CD44 signaling

Because HA is a known ligand of CD44, we investigated whether HA-mediated DMP-1 expression and odontoblastic differentiation arose via CD44 signaling. We precultured DPSCs with a CD44-neutralizing monoclonal antibody to inactivate CD44, and evaluated HA-induced DMP-1 expression levels. Inactivation of CD44 in DPSCs inhibited HA-induced DMP-1 expression (Fig. 7). This indicates that DMP-1 expression induced by HA involves CD44 signaling.

\section{Discussion}

We initially aimed to investigate the type of differentiation induced by CD44 stimulation in DPSCs. Our data show that CD44 was expressed in approximately $62 \%$ of DPSCs 
a

Phospho-p44/42

MAPK(Erk1/2)

beta-actin

$\mathrm{HA} \quad(10 \mu \mathrm{g} / \mathrm{ml})$

0

$\begin{array}{lll}5 & 15 & 30\end{array}$

60

180 (min)

b

Phospho-p44/42
MAPK(Erk1/2)

beta-actin

HA $(10 \mu \mathrm{g} / \mathrm{ml})$

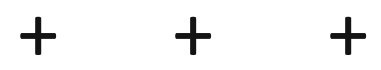

SCH772984(nM)

$\begin{array}{lll}- & 24\end{array}$

C

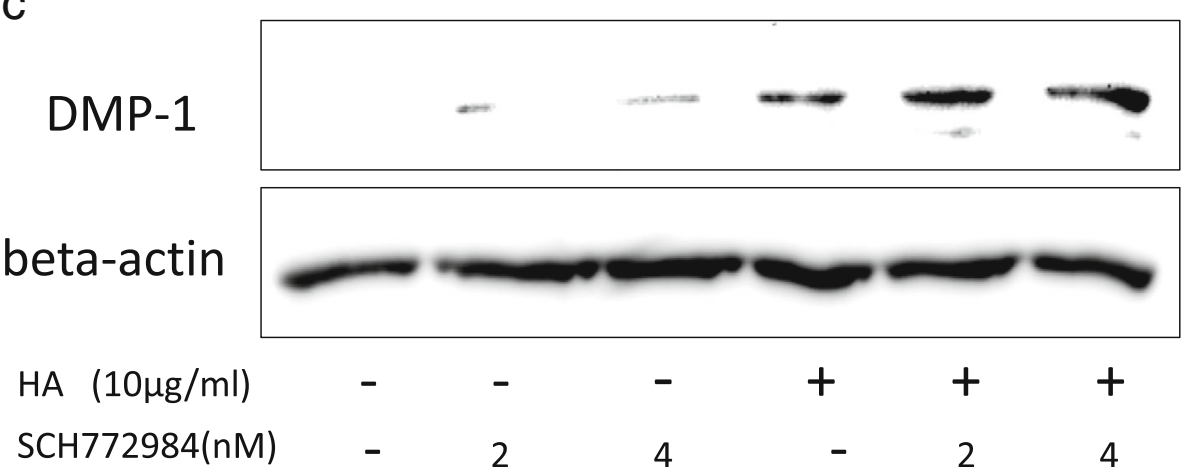

Fig. $6 \mathrm{HA}$ induces phosphorylation of Erk1/2 on DPSCs. a DPSCs were treated with HA (10 $\mu \mathrm{g} / \mathrm{mL})$ over a time course of 5-180 min and then examined for phosphorylation of Erk1/2 by western blotting. b DPSCs were treated with HA (10 $\mathrm{mg} / \mathrm{mL})$ in the presence of SCH772984 for $30 \mathrm{~min}$, and the inhibition of Erk1/2 phosphorylation was verified. c Following inhibition of Erk1/2 phosphorylation by SCH772984 treatment, DMP-1 expression was determined by western blotting. DMP-1 dentin matrix protein-1, HA hyaluronic acid, MAPK mitogen-activated protein kinase

and that odontoblastic differentiation was promoted by HA-induced stimulation of CD44 in DPSCs.

Previous studies have suggested that DPSCs have the potential to regenerate dental tissues [30], myoideum and nerve tissues $[9,31]$. Although dental pulp-derived induced pluripotent stem cells have been evaluated for use in clinical applications [32, 33], and HA stimulation of CD44 is important for differentiation leading to the production of odontoblasts, few studies have investigated the precise signaling mechanisms operating in DPSCs.

Low molecular weight HA can induce cell proliferation and induce osteocalcin mRNA expression in a dose-dependent manner in calvarial-derived mesenchymal cells [34]. However, others have reported that high molecular weight $\mathrm{HA}$ can induce mineralization of dental pulp tissue and dental pulp cells [35, 36]. Our investigation employed a high molecular weight 


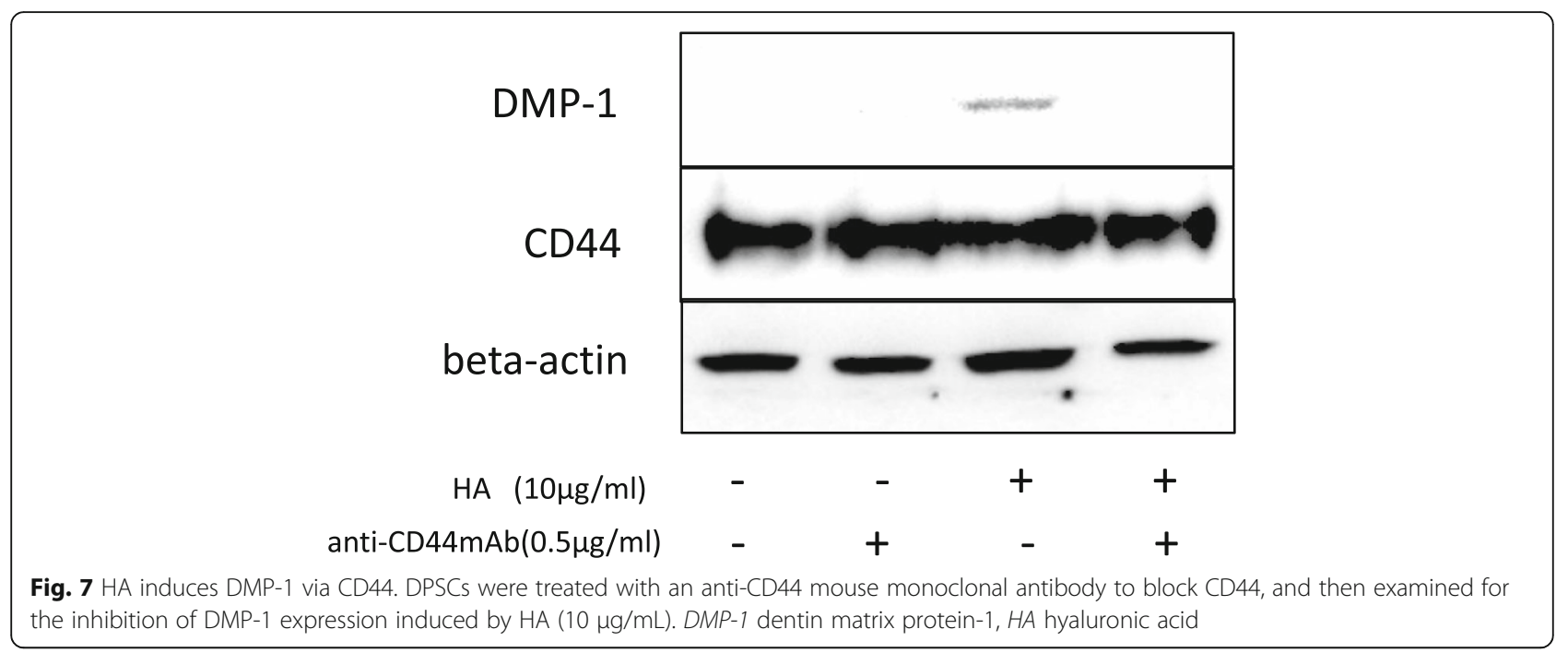

HA. Treatment with this high molecular weight HA increased the proportion of CD44-positive DPSCs from $62 \%$ to $72 \%$ at $5 \mathrm{~min}$ posttreatment. Thereafter, cell surface expression of $\mathrm{CD} 44$ declined to $22 \%$ at $24 \mathrm{~h}$ posttreatment (Fig. 1c). Meanwhile, CD44 levels in whole cell lysate remained unchanged following treatment with HA for either $24 \mathrm{~h}$ or 1 week (Fig. 2d and Fig. 7). These findings suggest that CD44 might shift into cells and away from the cell surface following HA treatment.

Although HA does not induce cell proliferation or affect the cell cycle in DPSCs (Fig. 2), we clearly demonstrated that HA signaling via CD44 is important for odontoblastic differentiation in DPSCs. Interestingly, HA induced activation of Smad1, Akt and Erk1/2, but not beta-catenin. The peak of Akt and Smad1 phosphorylation occurred $30 \mathrm{~min}$ after treatment with $\mathrm{HA}$, while the peak of Erk1/2 phosphorylation occurred 5 min after HA treatment. Furthermore, degradation of phosphorylated Erk1/2 did not occur as was the case for Akt and Smad1 phosphorylation (Figs. 4, 5, and 6). However, while HA-induced Erk1/2 activation exhibited different characteristics to HA-induced Akt and Smad1 activation, these signaling pathways did not directly promote odontoblastic differentiation in DPSCs. This suggests that there may be another as-yet unexplained signaling mechanism by which $\mathrm{HA}$ induces odontoblastic differentiation via CD44. Therefore, our present results and those of previous studies on HA treatment and dental pulp $[35,36]$ suggest that $\mathrm{HA}$ induces odontoblastic differentiation via CD44 signaling in DPSCs. Our findings indicate that the application of HA to dental pulp medicine may be useful for dental pulp capping or tooth regeneration in the future. Despite our best efforts, the mechanisms underlying CD44-induced differentiation of DPSCs to odontoblasts remain unclear, and will require further examination in future studies. Nevertheless, our present study describes an efficient differentiation method to derive odontoblasts from DPSCs.

\section{Conclusions}

HA induces odontoblastic differentiation of DPSCs via CD44, but does not promote cellular proliferation. While HA activates Akt, Smad and MAPK signaling, there is no clear relationship between these signaling pathways and the odontoblastic differentiation of DPSCs. These novel findings further our understanding of DPSC differentiation, and may facilitate advances in dental pulp therapy by enabling efficient induction of odontoblastic differentiation of DPSCs.

\section{Additional file}

Additional file 1: Figure S1. HA does not induce phosphorylation of beta-catenin on DPSCs. DPSCs were treated with HA $(10 \mu \mathrm{g} / \mathrm{mL})$ over a time course of 5-360 min and then examined for phosphorylation of beta-catenin by western blotting. (PPTX $1259 \mathrm{~kb}$ )

\section{Abbreviations}

ALP: alkaline phosphatase; BMP: bone morphogenetic protein; DMEM: Dulbecco's modified Eagle's medium; DMP-1: dentin matrix protein-1; DPSCs: dental pulp stem cells; DSPP: dentin sialophosphoprotein;

EDTA: ethylenediaminetetraacetic acid; FACS: fluorescence-activated cell sorting; FBS: fetal bovine serum; FITC: fluorescein isothiocyanate; HA: hyaluronic acid; IgG: immunoglobulin G; MAPK: mitogen-activated protein kinase; MTT: 3-(4,5-dimethylthiazol-2-yl)-2,5-diphenyltetrazolium bromide; PBS: phosphate-buffered saline; PMSF: phenylmethanesulfonyl fluoride

\section{Acknowledgements}

In addition, the authors wish to thank Mineko Tomomura PhD and Akito Tomomura PhD from the Division of Biochemistry, Meikai University, for their assistance in the preparation of this manuscript.

\section{Funding}

This investigation was supported in part by the Japan Society for the Promotion of Science Grants-in-Aid for Scientific Research (JSPS KAKENHI) 
(grant numbers 23592806, 24791982, 26462854, 26462892, and 26861748), and by research funds from Meikai University School of Dentistry in Japan.

\section{Availability of data and materials}

The authors give endorsement to share these data and materials in publicly available repositories.

\section{Authors' contributions}

NU contributed to experimental design, performed the majority of the experiments, data analysis, and drafted and critically revised the manuscript. EO performed some experiments, data analysis, participated in study design, and critically revised the manuscript. MJ performed some experiments, data analysis, and critically revised the manuscript. HK participated in study design, data analysis, and critically revised the manuscript. TK participated in study design, data analysis, manuscript preparation, and critically revised the manuscript. HS contributed to experimental conceptualization, data interpretation, and critically revised the manuscript. All authors read and approved the final manuscript.

\section{Authors' information}

NU was attached to the Division of Pharmacology, Department of Diagnostic and Therapeutic Sciences, Meikai University School of Dentistry in Japan from 2011 to 2015. During this time, he studied characteristic and differentiation induction of DPSCs with colleagues. He is presently continuing this work at the Department of Oral Biochemistry, Asahi University School of Dentistry, Japan. EO is a pharmacologist, and was attached to the Division of Pharmacology, Department of Diagnostic and Therapeutic Sciences, Meikai University School of Dentistry from 2013 to 2015. During this time, she studied the development of anticancer drugs and therapeutics involving human dental pulp. She is presently continuing this research at the Department of Natural Products Chemistry, Faculty of Pharmaceutical Science, Aomori University, in Japan.

\section{Competing interests}

The authors declare that they have no competing interests.

\section{Consent for publication}

Not applicable.

\section{Ethics approval and consent to participate}

Not applicable.

\section{Author details}

'Division of Pharmacology, Department of Diagnostic and Therapeutic Sciences, Meikai University School of Dentistry, 1-1 Keyakidai, Sakado, Saitama 350-0283, Japan. ${ }^{2}$ Department of Oral Biochemistry, Asahi University School of Dentistry, Gifu 501-0296, Japan. ${ }^{3}$ Department of Natural Products Chemistry, Faculty of Pharmaceutical Science, Aomori University, Aomori 030-0943, Japan. ${ }^{4}$ Division of Endodontics, Department of Restorative and Biomaterials Sciences, Meikai University School of Dentistry, Saitama 350-0283, Japan. ${ }^{5}$ Division of Operative Dentistry, Department of Diagnostic and Therapeutic Sciences, Meikai University School of Dentistry, Saitama 350-0283, Japan.

Received: 20 February 2016 Revised: 21 June 2016 Accepted: 30 August 2016 Published online: 20 September 2016

\section{References}

1. Smith AJ, Cassidy N, Perry H, Begue-Kirn C, Ruch JV, Lesot H. Reactionary dentinogenesis. Int J Dev Biol. 1995;39(1):273-80.

2. Gronthos S, Mankani M, Brahim J, Robey PG, Shi S. Postnatal human dental pulp stem cells (DPSCs) in vitro and in vivo. Proc Natl Acad Sci U S A. 2000;97(25):13625-30.

3. Kerkis I, Kerkis A, Dozortsev D, Stukart-Parsons GC, Gomes Massironi SM, Pereira LV, Caplan Al, Cerruti HF. Isolation and characterization of a population of immature dental pulp stem cells expressing OCT-4 and other embryonic stem cell markers. Cells Tissues Organs. 2006;184(3-4):105-16.

4. Beltrao-Braga PC, Pignatari GC, Maiorka PC, Oliveira NA, Lizier NF, Wenceslau CV, Miglino MA, Muotri AR, Kerkis I. Feeder-free derivation of induced pluripotent stem cells from human immature dental pulp stem cells. Cell Transplant. 2011;20(11-12):1707-19.
5. Gronthos S, Brahim J, Li W, Fisher LW, Cherman N, Boyde A, DenBesten P, Robey PG, Shi S. Stem cell properties of human dental pulp stem cells. J Dent Res. 2002:81(8):531-5.

6. Laino G, d'Aquino R, Graziano A, Lanza V, Carinci F, Naro F, Pirozzi G, Papaccio G. A new population of human adult dental pulp stem cells: a useful source of living autologous fibrous bone tissue (LAB). J Bone Miner Res. 2005;20(8):1394-402.

7. Arthur A, Rychkov G, Shi S, Koblar SA, Gronthos S. Adult human dental pulp stem cells differentiate toward functionally active neurons under appropriate environmental cues. Stem Cells. 2008;26(7):1787-95.

8. Bakopoulou A, Leyhausen G, Volk J, Papachristou E, Koidis P, Geurtsen W. Wnt/beta-catenin signaling regulates Dental Pulp Stem Cells' responses to pulp injury by resinous monomers. Dent Mater. 2015;31(5):542-55.

9. Mead B, Berry M, Logan A, Scott RA, Leadbeater W, Scheven BA. Stem cell treatment of degenerative eye disease. Stem Cell Res. 2015;14(3): 243-57.

10. Graziano A, d'Aquino R, Laino G, Papaccio G. Dental pulp stem cells: a promising tool for bone regeneration. Stem Cell Rev. 2008;4(1):21-6.

11. Casagrande L, Cordeiro MM, Nor SA, Nor JE. Dental pulp stem cells in regenerative dentistry. Odontology. 2011;99(1):1-7.

12. Chen $\mathrm{KL}$, Huang $Y Y$, Lung J, Yeh YY, Yuan K. CD44 is involved in mineralization of dental pulp cells. J Endod. 2013;39(3):351-6.

13. Cywes C, Wessels MR. Group A Streptococcus tissue invasion by CD44-mediated cell signalling. Nature. 2001;414(6864):648-52.

14. Wolny PM, Banerji S, Gounou C, Brisson AR, Day AJ, Jackson DG, Richter RP. Analysis of CD44-hyaluronan interactions in an artificial membrane system: insights into the distinct binding properties of high and low molecular weight hyaluronan. J Biol Chem. 2010;285(39):30170-80.

15. Liao HX, Lee DM, Levesque MC, Haynes BF. N-terminal and central regions of the human CD44 extracellular domain participate in cell surface hyaluronan binding. J Immunol. 1995;155(8):3938-45.

16. Scheller EL, Chang J, Wang CY. Wnt/beta-catenin inhibits dental pulp stem cell differentiation. J Dent Res. 2008;87(2):126-30.

17. Shirai M, Imanaka-Yoshida K, Schneider MD, Schwartz RJ, Morisaki T. T-box 2, a mediator of Bmp-Smad signaling, induced hyaluronan synthase 2 and Tgfbeta2 expression and endocardial cushion formation. Proc Natl Acad Sci U S A. 2009;106(44):18604-9.

18. Arakaki M, Ishikawa M, Nakamura T, Iwamoto T, Yamada A, Fukumoto E, Saito M, Otsu K, Harada H, Yamada Y, et al. Role of epithelial-stem cell interactions during dental cell differentiation. J Biol Chem. 2012;287(13):10590-601.

19. Iohara K, Nakashima M, Ito M, Ishikawa M, Nakasima A, Akamine A. Dentin regeneration by dental pulp stem cell therapy with recombinant human bone morphogenetic protein 2. J Dent Res. 2004;83(8):590-5.

20. Hayano S, Kurosaka H, Yanagita T, Kalus I, Milz F, Ishihara Y, Islam MN, Kawanabe N, Saito M, Kamioka H, et al. Roles of heparan sulfate sulfation in dentinogenesis. J Biol Chem. 2012;287(15):12217-29.

21. Ono M, Ohkouchi S, Kanehira M, Tode N, Kobayashi M, Ebina M, Nukiwa T, Irokawa T, Ogawa H, Akaike T, et al. Mesenchymal stem cells correct inappropriate epithelial-mesenchyme relation in pulmonary fibrosis using stanniocalcin-1. Mol Ther. 2015;23(3):549-60.

22. Song M, Chung W, Lee SJ, Kim E. Long-term outcome of the cases classified as successes based on short-term follow-up in endodontic microsurgery. J Endod. 2012;38(9):1192-6.

23. Li F, Song N, Tombran-Tink J, Niyibizi C. Pigment epithelium-derived factor enhances differentiation and mineral deposition of human mesenchymal stem cells. Stem Cells. 2013;31(12):2714-23.

24. Zhai YK, Guo XY, Ge BF, Zhen P, Ma XN, Zhou J, Ma HP, Xian CJ, Chen KM. Icariin stimulates the osteogenic differentiation of rat bone marrow stromal cells via activating the PI3K-AKT-eNOS-NO-cGMP-PKG. Bone. 2014;66:189-98.

25. Hao J, Ho JN, Lewis JA, Karim KA, Daniels RN, Gentry PR, Hopkins CR, Lindsley CW, Hong CC. In vivo structure-activity relationship study of dorsomorphin analogues identifies selective VEGF and BMP inhibitors. ACS Chem Biol. 2010;5(2):245-53.

26. Vigetti D, Viola M, Karousou E, Rizzi M, Moretto P, Genasetti A, Clerici M, Hascall VC, De Luca G, Passi A. Hyaluronan-CD44-ERK1/2 regulate human aortic smooth muscle cell motility during aging. J Biol Chem. 2008:283(7):4448-58.

27. Judd NP, Winkler AE, Murillo-Sauca O, Brotman JJ, Law JH, Lewis Jr JS, Dunn GP, Bui JD, Sunwoo JB, Uppaluri R. ERK1/2 regulation of CD44 modulates oral cancer aggressiveness. Cancer Res. 2012;72(1):365-74. 
28. Morris EJ, Jha S, Restaino CR, Dayananth P, Zhu H, Cooper A, Carr D, Deng $Y$, Jin W, Black S, et al. Discovery of a novel ERK inhibitor with activity in models of acquired resistance to BRAF and MEK inhibitors. Cancer Discov. 2013;3(7):742-50.

29. Chen J, Lan Y, Baek JA, Gao Y, Jiang R. Wnt/beta-catenin signaling plays an essential role in activation of odontogenic mesenchyme during early tooth development. Dev Biol. 2009;334(1):174-85.

30. Lee JH, Lee DS, Choung HW, Shon WJ, Seo BM, Lee EH, Cho JY, Park JC. Odontogenic differentiation of human dental pulp stem cells induced by preameloblast-derived factors. Biomaterials. 2011;32(36):9696-706.

31. Ishizaka R, Hayashi Y, lohara K, Sugiyama M, Murakami M, Yamamoto T, Fukuta O, Nakashima M. Stimulation of angiogenesis, neurogenesis and regeneration by side population cells from dental pulp. Biomaterials. 2013;34(8):1888-97.

32. Tamaoki N, Takahashi K, Tanaka T, Ichisaka T, Aoki H, Takeda-Kawaguchi T, lida K, Kunisada T, Shibata T, Yamanaka S, et al. Dental pulp cells for induced pluripotent stem cell banking. J Dent Res. 2010;89(8):773-8

33. Yoo CH, Na HJ, Lee DS, Heo SC, An Y, Cha J, Choi C, Kim JH, Park JC, Cho YS. Endothelial progenitor cells from human dental pulp-derived iPS cells as a therapeutic target for ischemic vascular diseases. Biomaterials. 2013;34(33):8149-60.

34. Huang $L$, Cheng YY, Koo PL, Lee KM, Qin L, Cheng JC, Kumta SM. The effect of hyaluronan on osteoblast proliferation and differentiation in rat calvarialderived cell cultures. J Biomed Mater Res A. 2003;66(4):880-4.

35. Sasaki T, Kawamata-Kido H. Providing an environment for reparative dentine induction in amputated rat molar pulp by high molecular-weight hyaluronic acid. Arch Oral Biol. 1995;40(3):209-19.

36. Chen KL, Yeh YY, Lung J, Yang YC, Yuan K. Mineralization effect of hyaluronan on dental pulp cells via CD44. J Endod. 2016;42(5):711-6. 